\title{
Dual-core Optical Fiber as Beam Splitter with Arbitrary, Tunable Polarization-dependent Transfer Function
}

\author{
Nina Podoliak and Peter Horak
}

\begin{abstract}
We present the design of a microstructured dualcore optical fiber with integrated electrodes and filled with liquid crystals. The dual-core structure acts as a directional coupler whose properties depend on the liquid crystal alignment. We show that with four electrodes and two separate driving voltages below $30 \mathrm{~V}$ on the electrodes, the beam-splitting properties of the fiber can be controlled independently and continuously for the two polarization components, thus allowing for the realization of any arbitrary $2 \times 2$ transfer function, such as tunable polarizers, polarization-dependent attenuators, or polarization-independent beam splitting.
\end{abstract}

Index Terms-Directional couplers, Liquid crystals, Optical fiber devices

\section{INTRODUCTION}

$\mathrm{P}$ OLARIZATION beam splitters, filters and attenuators are essential optical components for various applications such as polarization-division multiplexing in optical telecommunication networks [1], or polarization encoding in quantum information systems [2], [3]. Splitting between the TE- and TM-polarized modes of optical waveguides has been demonstrated by utilizing different photonic elements, including Mach-Zehnder interferometers [4], multi-mode interference devices [5], [6], directional couplers [3], [7] or waveguide grating structures [8]. Among them, the directional coupler is one of the most widely used structures to achieve a high polarization extinction ratio. It consists of two distinct waveguides placed close together and coupled through overlapping evanescent fields of propagating modes. The coupling strength can be precisely engineered by controlling the waveguide geometry and the length of the interaction region. To some limited degree, post-fabrication tuning can also be achieved by changing either the propagation constants of optical modes or the separation between waveguides.

This work was supported through the U.K. National Quantum Technology Programme (EPSRC grants EP/M013243/1 and EP/M013294/1).

N. Podoliak is with Physics and Astronomy, University of Southampton, Southampton SO17 1BJ, U.K. (e-mail: n.podoliak@soton.ac.uk).

P. Horak is with the Optoelectronics Research Centre, University of Southampton, Southampton SO17 1BJ, U.K. (e-mail: peh@orc.soton.ac.uk).

Color versions of one or more of the figures in this paper are available online at http://ieeexplore.ieee.org.

Digital Object Identifier: 10.1109/JLT.2017.2729939
Polarization beam splitters based on directional couplers can in principle be realized using different waveguide geometries and fabrication platforms. However, most of them so far were fabricated using planar waveguide structures on integrated photonic chips [3], [7], [9]. On the other hand, the realization of directional couplers with polarizing, and ideally continuously tunable, functionality in a fiber platform [10][13] would allow for a new generation of all-fiber photonic devices in particular for telecommunication, quantum information, and sensing applications.

The possibility of selective filling of air holes in microstructured fibers with different materials such as gases or liquids enables on-demand modification of fiber properties. The most well-known and widely-used optical materials for controlling and switching of light are liquid crystals (LCs). LCs are highly birefringent materials, the properties of which can be controlled by electric or magnetic fields, temperature or light. Polarization control [14] and selective polarization switching [15]-[19] were demonstrated using standard photonic crystal fibers with holes containing LCs. An electrooptic polarization switch was also demonstrated in dual-core photonic crystal fiber structures, one [11] or both [12] of the cores of which were filled with LCs. By reorientation of LC alignment in the fiber core, matching of propagation constants between the two cores was achieved yielding high contrast polarization splitting. The drawback of these dual-core fibers is that light is guided in the LC filled core causing high optical losses due to scattering by LC molecular fluctuations. Moreover, reorientation of LC in the small fiber holes was achieved by applying voltages to macroscopic electrodes mounted outside the fiber far away from the core structure. Thus, the driving voltage for these fibers was large, about 100$200 \mathrm{~V}$.

Here we propose a novel design of a widely tunable microstructured dual-core optical fiber with LC cladding and integrated electrodes. The proposed fiber design has the following advantages. Firstly, the fiber has a dual-core glass structure, which acts as a polarization sensitive directional coupler. Such fibers have been fabricated previously and optical switching was investigated by either Joule heating [20], or mechanical movement of the cores by gas pressure [10], and electrostatic forces [21]. Secondly, we choose high refractive index lead-silicate glasses as a fiber material with a refractive index larger than the index of typical LCs, allowing 


\section{JOURNAL OF LIGHTWAVE TECHNOLOGY}

for index-guided optical modes confined in a low-loss glass core rather than inside the LCs, which leads to significantly lower propagation losses [17], [22]. In contrast to photonic crystal fibers, the LC filled holes in the proposed fiber design are much larger in size, which greatly facilitates LC reorientation. Moreover, embedding conducting electrodes inside the fiber cladding as demonstrated in [20] significantly reduces the driving voltages required for LC alignment, allowing for low energy consumption. In particular, we propose a design with four electrodes embedded in the cladding which gives freedom to control the orientation of the electric field and hence LC alignment in any direction inside the fiber. Having electrodes integrated in the cladding also makes the fiber devices compact and truly integrated.

\section{FiBer STRUCTURE AND MOdELING}

The fiber design is shown in Fig. 1 (a). The fiber consists of two elongated glass cores, which we model by elliptical shapes of $1.5 \times 3 \mu \mathrm{m}$ size, that are independently supported by two $0.5 \mu \mathrm{m}$ thick and $15 \mu \mathrm{m}$ long glass membranes. These dimensions are similar to those of a previously fabricated fiber [20], which was shown to be stiff enough to eliminate any core displacement or vibrations [23]. The cores are separated by a small gap, uniform along the fiber length. The central hole around the cores is $30 \mu \mathrm{m}$ in diameter and filled with LCs. There are four metal electrodes embedded in the glass cladding (as shown in Fig. 1 (a)). The electrode diameter is 50 $\mu \mathrm{m}$ and their edges are $25 \mu \mathrm{m}$ away from the center of the fiber. Driving AC voltages, with RMS values of $V_{1}$ and $V_{2}$, are applied between pairs of opposite electrodes with the polarity shown in Fig. 1 (a).

Design and simulation of the fiber was performed using advanced optical modal simulations, based on a full-vector finite element method implemented in Comsol Multiphysics ${ }^{\circledR}$, in combination with a custom-made LC model [24] based on the Frank-Oseen continuous elastic theory approach [25]. LC alignment is described by a director $\boldsymbol{l}$ defined by the tilt and twist angles, $\theta$ and $\varphi$, as shown in Fig. 1(a). As the first step, we simulate both the spatial distribution of the control electric field created between electrodes and the induced LC director alignment, which are coupled through a LC-dielectric tensor. The LC optical dielectric tensor, defined as $\varepsilon_{i j}=n_{o}{ }^{2}+\left(n_{e}{ }^{2}-n_{o}{ }^{2}\right) l_{i} l_{j}$ where $n_{o}$ and $n_{e}$ are the ordinary and extraordinary LC refractive indices, is then used in the optical modal simulation. The eigenmode propagation constants are used to calculate the switching of light between the fiber cores at the output.

We choose a high-index lead silicate glass as fiber material, Schott SF57, with a refractive index of 1.8 at $1550 \mathrm{~nm}$ wavelength. This refractive index is higher than refractive indices of typical nematic LCs, which allows for index guiding inside the glass cores surrounded by the LC cladding. Specifically, we assume the well-studied nematic LC E7 with ordinary and extraordinary refractive indices of 1.5 and 1.7 at $1550 \mathrm{~nm}$ wavelength, respectively. It has a positive dielectric anisotropy and thus tends to align along the electric field lines in an external electric field. The values of splay, twist and bend LC elastic constants of $11.2 \mathrm{pN}, 6.8 \mathrm{pN}$ and $17.8 \mathrm{pN}$, and the low frequency dielectric constants of 14.2 and 5.1 are used in the LC simulation. We emphasize that while we choose specific fiber dimensions and materials for the following simulations, our findings are indeed general and analogous results can be achieved with a wide range of fiber parameters.

\section{OPtiCAL PROPERTIES OF DUAL-CORE FibER WITH LC ClADDING}

As the optical cores in the fiber are placed close to each other, the individual core modes couple through their overlapping evanescent fields, which stretch into the LC medium, forming symmetric and antisymmetric superposition modes (supermodes) shown in Fig. 1 (b). We found that at the simulated fibre parameters the constructive and destructive interference of the supermodes yields the optical power being fully localized in one of the optical cores. Launching light into a single core of the fiber thus excites a combination of symmetric and antisymmetric supermodes and causes the optical power to oscillate between the two cores upon propagation with the period (beat length) defined as

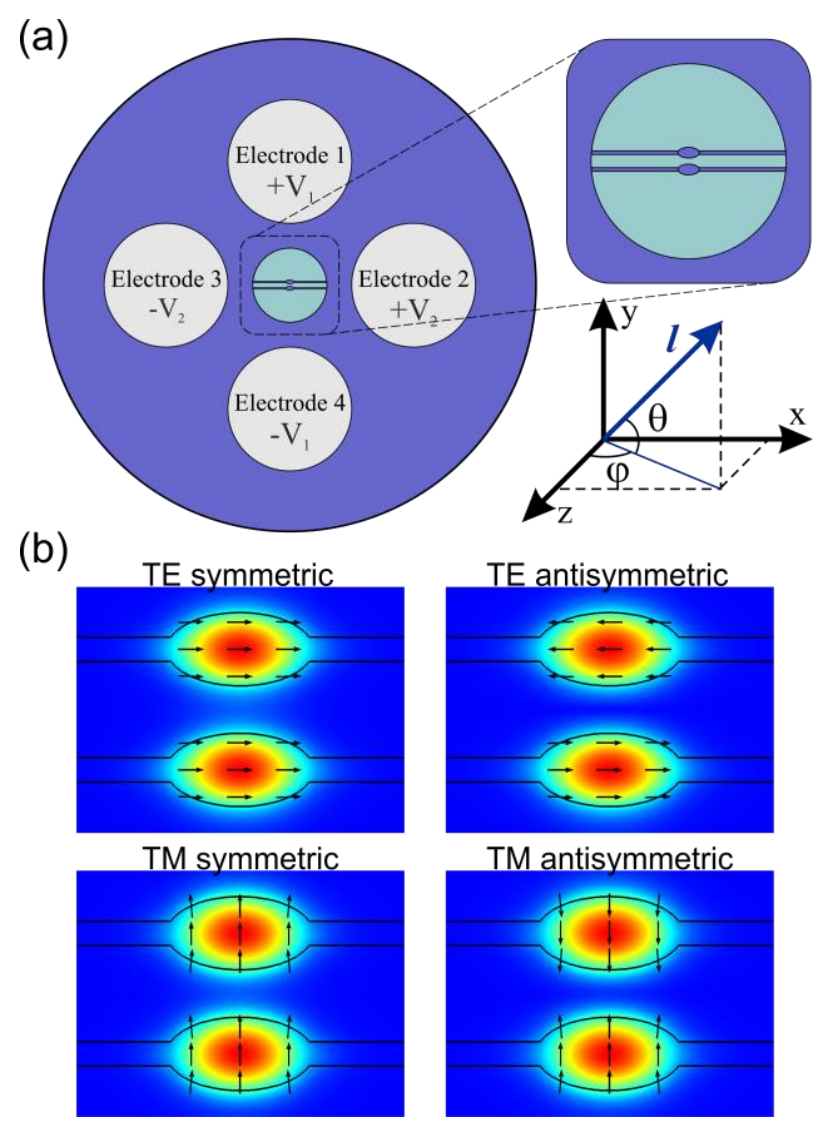

Fig. 1. (a) Geometry of the dual-core fiber with electrodes integrated in the cladding. The inset is a magnification of the central part of the fiber. The light blue color marks the central fiber holes filled with LCs. LC director alignment $\boldsymbol{l}$ is described by the tilt and twist angles, $\theta$ and $\varphi$. (b) Normalized optical mode profile of TE and TM-like symmetric and antisymmetric supermodes in the voltage-off state. Black arrows show the polarization direction. 


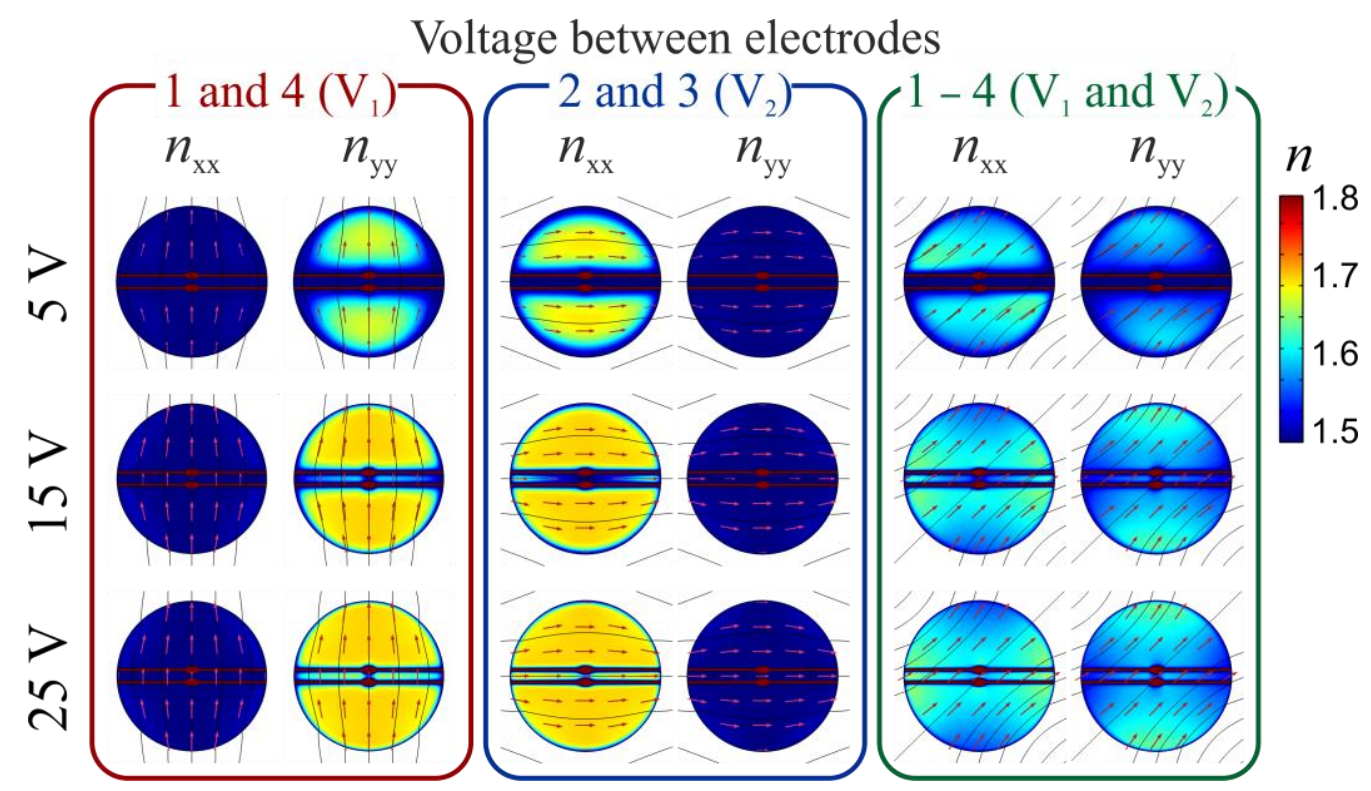

Fig. 2. Colormaps showing the profiles of the refractive index tensor ( $n_{x x}$ and $n_{y y}$ components) around the central structure of the fiber at different voltages applied between different sets of electrodes. The set of driving electrodes is given on the top of each column and the RMS voltage value is given in the left column. Red arrows show LC alignment and hence the direction of optical axis and black lines mark the electric field direction.

$L_{b}=\lambda /\left(n_{s}-n_{a}\right)$, where $\lambda$ is the light wavelength, and $n_{s}$ and

$n_{a}$ are the effective mode indices of the symmetric and antisymmetric supermodes, respectively. Due to the elliptical shape, the guiding cores are birefringent with two distinct fundamental guiding modes: TE- and TM-like modes with predominant $x$ - and $y$-polarization, respectively (see Fig. 1 (b)). The propagation constants, and hence the beat lengths of different polarization supermodes are highly dependent on the size of the gap between the cores as well as on the refractive index of the medium around the cores. Varying the mode propagation constants and hence the beat length causes continuous switching of light between the two cores at the fiber output. It should be noted that the full switching can only be achieved if the two cores are identical and equal amplitudes of symmetric and antisymmetric supermodes are coupled into the fibre. In our previous studies, we demonstrated optical switching in the dual-core fiber by variation of the gap between the cores by applying air pressure [10] or generating electrostatic forces between the cores [21]. Here, we investigate changing the fiber properties by modifying the cladding refractive index through alignment of LCs. As we will show below, this allows for much more versatile functionalities and more stable operation. We should point out that, in contrast to our previous study [21], no electrostatically induced core displacement is expected in the fiber discussed here. While the fiber design is similar, the polarity of driving voltages is different. The voltage polarity shown in Fig. 1 (a) creates a uniform electric field in the center around the cores with negligible gradient, causing no electrostatic forces between the cores [21].

The initial alignment of LC molecules inside the fiber holes for zero applied voltage is along the fiber axis (z-axis). The axial LC alignment is typical for small capillaries. It was observed in holes of photonic crystal fibers by filling them with LC in the isotropic state and slowly cooling down [11], [17]. This spontaneously induced alignment can be further improved by coating the inner glass surfaces with surfactants $[15,16]$. This corresponds to the boundary conditions for the tilt and twist LC angles of $\theta=\varphi=0$ on the glass surfaces. In the initial state, both $n_{x x}$ and $n_{y y}$ components of the LC refractive index tensor are equal to the ordinary index of LC. Driving voltage applied to the electrodes creates an electric field in the $(x, y)$-plane which tends to realign LC molecules. The four-electrode configuration of Fig. 1 (a) gives the flexibility to control LC alignment in any direction in the transverse $(x, y)$-plane.

Figure 2 shows the variation of the $n_{x x}$ and $n_{y y}$ components of the LC refractive index tensor around the fiber cores at driving voltages applied between different pairs of electrodes (electrode marking and voltage polarity are shown in Fig. 1 (a)). When the voltage is applied between electrodes 1 and 4, the generated electric field is predominantly directed along the $y$-axis, reorienting LCs inside the fiber along the same direction. This causes changes of the $n_{y y}$ component of the refractive index tensor while the $n_{x x}$ component remains unchanged. Likewise, the application of voltages between electrodes 2 and 3 reorients LCs along the $x$-axis, causing mainly the $n_{x x}$ component of the refractive index to change. Applying voltages to all electrodes reorients LC at an angle changing both $n_{x x}$ and $n_{y y}$. Moreover, by changing the ratio between voltage amplitudes $\mathrm{V}_{1}$ and $\mathrm{V}_{2}$, it is possible to rotate the electric field and hence achieve LC alignment at any angle in the $(x, y)$-plane. 
(a)

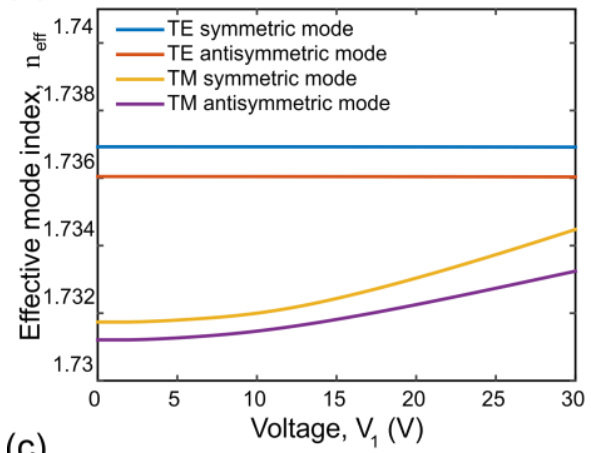

(c)

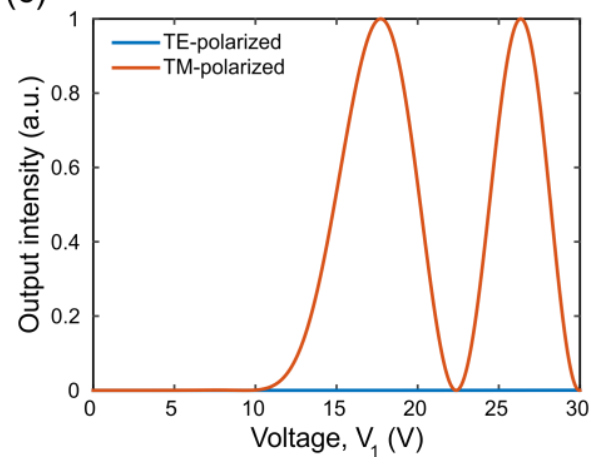

(b)

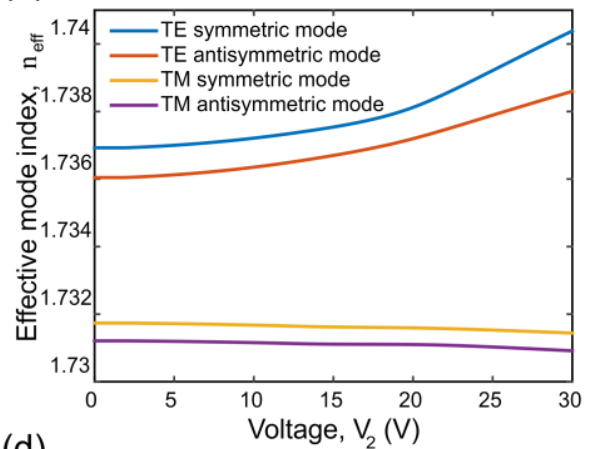

(d)

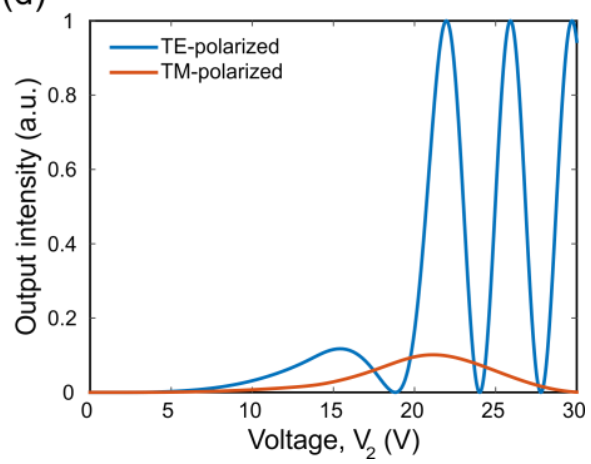

Fig. 3. (a), (b) Variation of the effective indices of the four fundamental fiber supermodes. (c), (d) Normalized intensities of different polarized components at the output port depending on the voltages applied between electrodes 1 and $4\left(\mathrm{~V}_{1}\right)$ or 2 and $3\left(\mathrm{~V}_{2}\right)$, respectively. Fiber parameters are given in the main text.

\section{DEVICE PERFORMANCE}

\section{A. Selective Polarization Switching with One Control Voltage}

The selective variation of the $n_{x x}$ or $n_{y y}$ components of the refractive index around the fiber cores predominantly modifies the propagation constants of only particularly polarized supermodes, as shown in Fig. 3 (a) and (b). Application of voltages between electrodes 1 and $4\left(\mathrm{~V}_{1}\right)$ changes the effective indices of the $y$-polarized TM supermodes, while the indices of the $x$-polarized TE supermodes remain unchanged. In a similar way, application of voltages between electrodes 2 and $3\left(\mathrm{~V}_{2}\right)$ changes mainly the effective indices of the TE supermodes. However, slight variations of the TM-mode indices are also observed in this case. Even while the TMsupermode is predominantly $y$-polarized, it also contains a small fraction of $x$-polarization around the boundary due to the elliptical shape of the cores (see Fig. 1 (b)), making it slightly sensitive to variations of the $n_{x x}$ component of the LC refractive index.

If a combination of symmetric and antisymmetric supermodes is exited in the fiber, meaning that the light is coupled into one of the cores of such a fiber (input port), selective switching of LCs modifies the beat length of the particular polarization component of the propagating beam, leading to polarization dependent switching of the output light from this core (output port) to the other core (drop port). As shown in our previous study, the beat length between two cores of a dual-core fiber is highly sensitive to the size of the gap between the cores [21]. For an effective performance as a tunable polarization splitter, we optimize the gap between the cores to obtain an initial "dark" state in the voltage-off case with undisturbed LC cladding, i. e., light of both polarization couples from the input port to the drop port. This optimal gap size is $0.97 \mu \mathrm{m}$ for the fiber parameters under consideration, at which the beat lengths between the TE- and TM-supermodes in the voltage-off state are $1.76 \mathrm{~mm}$ and $2.93 \mathrm{~mm}$, respectively. Thus, for a $4.4 \mathrm{~mm}$ long piece of fiber, the TEand TM-supermodes undergo 2.5 and 1.5 beat cycles along the fiber length, respectively, resulting in a high contrast initial dark state.

Using the mode effective indices, we calculate the fraction of light with TE- or TM-polarization coupled from input port to output port (normalized output intensity). Figure 3 (c) and (d) show the variation of the normalized intensity of the TE$\left(\mathrm{I}_{\mathrm{x}}\right)$ and TM-polarized $\left(\mathrm{I}_{\mathrm{y}}\right)$ components at the output port depending on the voltages applied between different sets of electrodes. As we only consider the coupling of light between two cores, the corresponding normalized intensity at the drop port is $\left(1-\mathrm{I}_{\mathrm{x} / \mathrm{y}}\right)$. When voltage is applied between electrodes 1 and 4, there is no TE-polarized component at the output port (it fully couples to the drop port), while the intensity of the TM-component varies with increasing driving voltages. At a voltage of $17.7 \mathrm{~V}$, the fiber acts as a polarization beam splitter: the TM-polarized component of the input beam appears at the output port while the TE-polarized component is fully coupled to the drop port with an extinction ratio between the two polarizations of $47 \mathrm{~dB}$, defined as the ratio between the output intensities of the two orthogonal polarization components. Moreover, by varying the driving voltage from 0 to $17.7 \mathrm{~V}$, gradual tuning of the TM-polarized beam intensity at the 
(a)

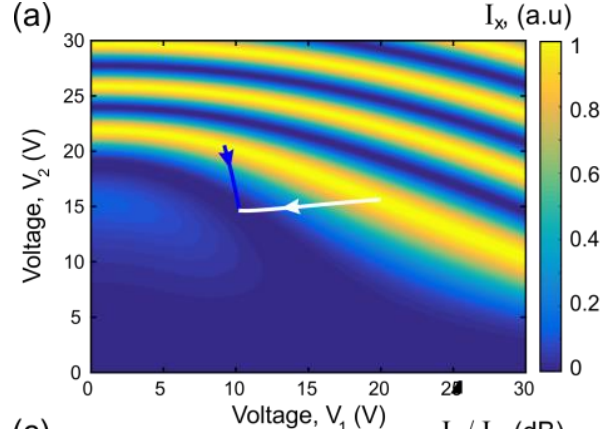

(c)

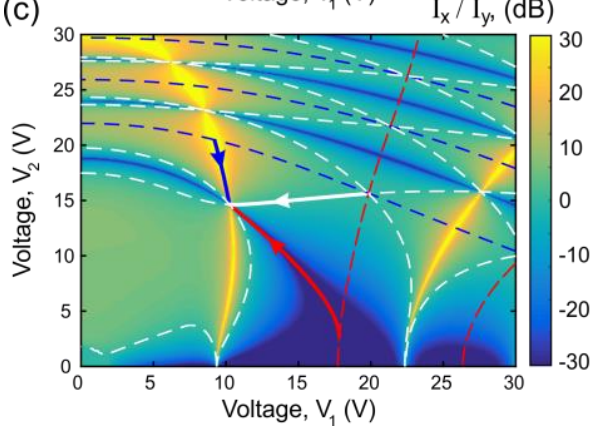

(b)
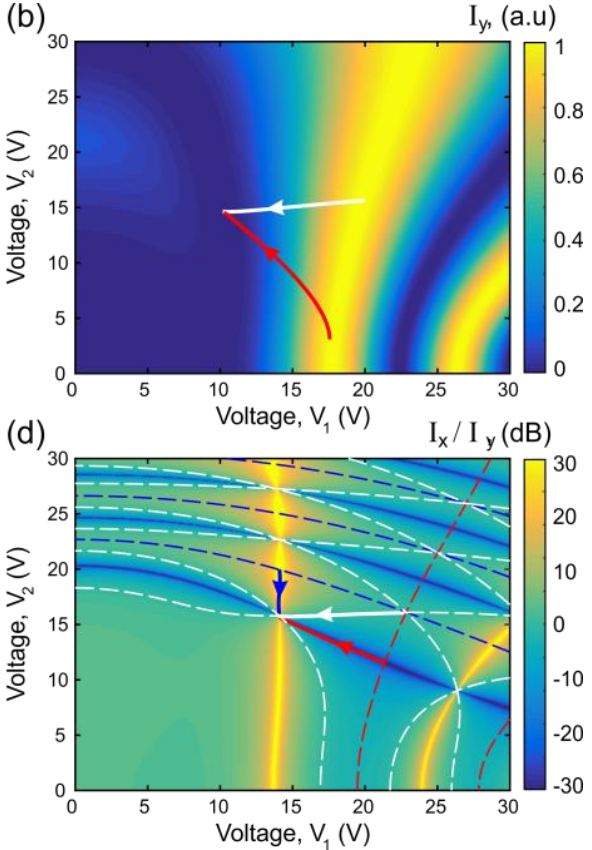

Fig. 4. (a), (b) Normalized intensities of TE- $\left(\mathrm{I}_{x}\right)$ and TM-polarized $\left(\mathrm{I}_{y}\right)$ components of the output beam depending on the applied voltages $\mathrm{V}_{1}$ and $\mathrm{V}_{2}$. (c) Polarization extinction ratio $\mathrm{I}_{x} / \mathrm{I}_{y}$ in decibel scale. Bright yellow and deep blue regions mark areas of TE- and TM-polarized output with extinction ratio $>30$ dB. Dashed blue, red, and white lines mark sets of driving voltages where $\mathrm{I}_{x}=1, \mathrm{I}_{y}=1$, or $\mathrm{I}_{x}=\mathrm{I}_{y}$, respectively. Thick blue and red lines are examples of driving voltages where the fiber operates as an attenuator for TE- and TM-polarized light, respectively. Along the thick white line the fiber operates as a polarization insensitive attenuator (see text for details). In (a)-(c) we assume unpolarized light coupled to the input core of a 4.4 mm long piece of the fiber with optimized gap between the cores of $0.97 \mu \mathrm{m}$. (d) Polarization extinction ratio $\mathrm{I}_{x} / \mathrm{I}_{y}$ equivalent to (c) calculated for a $4.4 \mathrm{~mm}$ long piece of the fiber with a non-optimized gap of $1 \mu \mathrm{m}$.

output is achieved. The opposite polarization switching is observed at around 29.7 V applied between electrodes 2 and 3. However, the extinction ratio in this case is lower, $25 \mathrm{~dB}$, and a small component of TM mode is present in the output port at a lower voltage because of the small sensitivity of the TM mode to a change of the $n_{x x}$ component of the cladding refractive index, as discussed above.

\section{B. Polarization Beam Splitter with Tunable Transfer Function}

The selective polarization switch shown in Fig. 3 requires precise control of the fiber parameters, such as fiber length and coupling strength between the cores (gap between cores), as it relies on the initial dark state for both polarization components in the voltage-off case. More diverse and precise fiber performance can be achieved when driving voltages $V_{1}$ and $\mathrm{V}_{2}$ are applied between both pairs of electrodes simultaneously. Fig. 4 (a) and (b) show the normalized intensities of TE- $\left(\mathrm{I}_{x}\right)$ and TM-polarized $\left(\mathrm{I}_{y}\right)$ components at the output port depending on driving voltages $V_{1}$ and $V_{2}$. As expected, intensity $I_{x}$ mainly varies with voltage $V_{2}$, although some dependence on voltage $V_{1}$ is also observed. There is a similar dependence of $\mathrm{I}_{\mathrm{y}}$ on $\mathrm{V}_{1}$. The combination of driving voltages $V_{1}$ and $V_{2}$ allows for versatile fiber functionality as a tunable polarizer, an attenuator, or a polarization-independent tunable beam splitter as discussed in the following.

Using intensities $\mathrm{I}_{x}$ and $\mathrm{I}_{y}$, we calculate the polarization extinction ratio of light at the output port (ratio $\mathrm{I}_{x} / \mathrm{I}_{y}$ shown in Fig. 4 (c)). Fig 4(c) is used to find a range of driving voltages at which a particular polarization of output light is achieved.
Bright yellow and deep blue regions mark areas where the output beam is fully TE- or TM-polarized with extinction ratios $>30 \mathrm{~dB}$. Dashed blue and red lines mark sets of driving voltages where the maximum of intensities $\mathrm{I}_{x}$ and $\mathrm{I}_{y}$ are observed, respectively, meaning that the whole TE- or TMpolarized component of the input beam is coupled to the output port. The fiber thus operates as a polarization beam splitter for a set of driving voltages obtained from the intersection of the dashed blue lines with the bright yellow regions. In these cases, the TE- and TM-components of the input beam are fully split between the output and drop ports. The opposite polarization splitting can be achieved for a set of driving voltages lying on the intersection of the dashed red lines with the deep blue regions.

Apart from polarization beam splitting, the fiber can demonstrate functionalities that are more diverse. One example is an all-fiber polarizer with variable attenuation. By varying the driving voltages from inside the regions where the output beam is fully polarized, as for example along the thick blue and red lines shown in Fig. 4 (a)-(c), the intensity of the output beam with the particular polarization can be gradually tuned form 1 to 0 . A polarization insensitive beam splitter can also be constructed based on this fiber design. Dashed white lines in Fig. 4(c) mark a set of driving voltages at which the intensities $\mathrm{I}_{x}$ and $\mathrm{I}_{y}$ are equal, meaning that both the output port and the drop port maintain the input polarization. By varying the driving voltages $V_{1}$ and $V_{2}$ along these paths, e.g. along the thick white line in Fig. 4 (a)-(c), an all-fiber beam splitter with arbitrary splitting ratio, but identical for the TEand TM-polarizations, can be achieved. 


\section{JOURNAL OF LIGHTWAVE TECHNOLOGY}

\section{Discussion of Device Fabrication and Calibration}

Figure 4(d) shows the polarization extinction ratio calculated for a $4.4 \mathrm{~mm}$ long piece of the fiber with a nonoptimized gap between the optical cores of $1 \mu \mathrm{m}$. At this gap size there is no initial dark state at the output port in the voltage off case. In this case, it is not possible to achieve a high aspect ratio polarization splitting by applying voltages between only one pair of electrodes, as indicated by the absence of the deep blue region at $\mathrm{V}_{2}=0$. However, using a combination of both $\mathrm{V}_{1}$ and $\mathrm{V}_{2}$, a suitable set of driving voltages can be selected for the same functionality as in the case of the fiber with optimized gap size (compare Fig. 4(c) and (d)). Similar corrections to the driving voltages can be obtained if the operational length of the fiber differs from the designed one. Thus, although the fiber performance is highly sensitive to the structural dimensions, fabricated inaccuracies in fiber dimensions can be corrected by calibrating the driving voltages.

The presented fiber is designed for operation at $1550 \mathrm{~nm}$ wavelength. To estimate the operational bandwidth of the device, we calculated the variation of effective mode indices with wavelength in the voltage-off case or at a fixed voltage. The material dispersion for the glass and liquid crystal was taken into account. We found that the operational bandwidth is limited to $\sim 10 \mathrm{~nm}$ mainly by the modal dispersion. However, the same piece of fiber can be easily calibrated for operation at another working wavelength by choosing a different set of driving voltages as described above.

In our previous works, we reported on the fabrication of dual suspended core fiber using an extrusion method to produce a glass preform, followed by a co-draw technique from the preform where some glass capillaries are filled with tin to form the electrodes [10], [20]. An optical loss of the order of $2-3 \mathrm{~dB} / \mathrm{m}$ was measured in such a fiber [10]. To construct a tunable beam-splitting device as discussed here, a piece of the fiber can be filled by LCs using capillary forces. The active length of the fiber can be controlled during the LC filling process assisted by a controlled pressure gradient. If encapsulation is required, UV curable polymers can be used to seal the LC filled section of the fiber. Additional scattering losses by the LC material around the fiber cores are expected to be not more than a few $\mathrm{dB} / \mathrm{m}$ [22]. Moreover, only a few $\mathrm{mm}$ active length of the fiber containing LCs is required for a device, which should bring the losses connected with the LC cladding to a minimum.

For a practical device realization, a piece of the dual-core fiber should be spliced to a standard single-mode fiber. It is worth reminding that the proposed device operation strongly relays on excitation of symmetric and antisymmetric supermodes with equal amplitudes at the fiber input, which might be an engineering challenge. Due to the mode size mismatch, a dual-core taper that converts the mode size of each core to dimensions matching standard fiber will be required at the output. At the input, however, coupling can be achieved by an intermediate section of fiber containing only a single suspended core, which can be butt-coupled to a single mode fiber [10]. There is a possibility of optical coupling between the cores outside the LC filled region of the fiber or in the taper. However, this unwanted coupling will have a constant strength, and hence can be corrected by postcalibrating the set or driving voltages after assembling as described above. Finally, the integrated electrodes can be accessed by selective etching holes in the glass cladding.

Another important characteristic is the switching speed of the fiber. LC reorientation time in a layer of thickness $d$ can be estimated as: $\tau=\frac{\tau_{0}}{\left(V / V_{t h}\right)^{2}-1}$, where $\tau_{0}=\frac{\gamma d^{2}}{\pi^{2} K}, \gamma$ is the rotation viscosity, $K$ is the elastic constant and $V_{t h}$ is the LC reorientation threshold (Frederiks threshold) [25]. For a $30 \mu \mathrm{m}$ thick LC E7 layer, $\tau_{0} \approx 2 \mathrm{~s}$. The operation voltage of the proposed fiber device is around 20 Volts, while the LC Frederiks threshold in this fiber is $\sim 2$ Volts, making the LC reorientation time in the cladding of the order $20 \mathrm{~ms}$.

\section{CONCLUSION}

In summary, we proposed a widely tunable microstructured dual-core optical fiber with LC cladding and integrated electrodes. The dual-core structure operates as a directional coupler, the propagation properties of which can be tuned by realigning the LCs in the fiber cladding with voltages applied to the electrodes. The fiber can be fabricated from a high refractive index lead-silicate glass which leads to light confinement predominantly in the glass cores for typical LCs, thereby ensuring low propagation losses. Embedding electrodes in the glass cladding allows fiber operation at low driving voltages below $30 \mathrm{~V}$. The four-electrode design with two independent driving voltages enables versatile control of the LC alignment inside the fiber and hence a wide tunability of the output beam intensity and polarization state. In principle, the fiber acts as a $2 \times 2$ beam splitter whose properties can be simultaneously and independently chosen for the two polarization components. In particular, we demonstrated that by selecting the correct set of driving voltages the same piece of fiber can operate as an all-fiber switchable polarization beam splitter, a polarizer with variable attenuation, or a polarization insensitive tunable beam splitter.

\section{ACKNOWLEDGMENT}

N. Podoliak would like to thank O. Buchnev for stimulating discussions. The data reported in this work is accessible through the University of Southampton institutional repository http://dx.doi.org/10.5258/SOTON/403786.

\section{REFERENCES}

[1]E. F. Mateo, X. Zhou, and G. Li, "Improved digital backward propagation for the compensation of inter-channel nonlinear effects in polarizationmultiplexed WDM systems," Opt. Express, vol.19, no. 2, pp. 570-582, 2011.

[2]C.-Z. Peng, J. Zhang, D. Yang, W.-B. Gao, H.-X. Ma, H. Yin, H.-P. Zeng, T. Yang, X.-B. Wang, and J.-W. Pan, "Experimental long-distance decoy-state quantum key distribution based on polarization encoding", Phys. Rev. Lett., vol. 98, p. 010505, 2007.

[3]A. Crespi, R. Ramponi, R. Osellame, L. Sansoni, I. Bongioanni, F. Sciarrino, G. Vallone and P. Mataloni, "Integrated photonic quantum gates for polarization qubits”, Nat. Commun., vol. 2, p. 566, 2011. 


\section{JOURNAL OF LIGHTWAVE TECHNOLOGY}

[4] Y. Hashizume, T. Goh, Y. Inoue, K. Hamamoto, and M. Itoh, "Polarization beam splitter with different core widths and its application to dualpolarization optical hybrid", J. Lightwave Technol., vol. 33, no. 2, pp. 408-414, 2015.

[5]X. Guan, H. Wu, Y. Shi, and D. Dai, "Extremely small polarization beam splitter based on a multimode interference coupler with a silicon hybrid plasmonic waveguide," Opt. Lett., vol. 39, no. 2, pp. 259-262, 2014.

[6]M. Yin, W. Yang, Y. Li, X. Wang, H. Li, "CMOS-compatible and fabrication-tolerant MMI-based polarization beam splitter, " Opt. Commun, vol.. 335, pp. 48-53, 2015.

[7]I. Kiyat , A. Aydinliand and N. Dagli, "A compact silicon-on-insulator polarization splitter," IEEE Phot. Technol. Lett., vol. 17, no.1, pp. 100102, 2005.

[8] K. Zhou, G. Simpson, X. Chen, L. Zhang, and I. Bennion, "High extinction ratio in-fiber polarizers based on $45^{\circ}$ tilted fiber Bragg gratings," Opt. Lett., vol. 30, no. 11, pp. 1285-1287, 2005.

[9]X. Sun, M. Z. Alam, J. S. Aitchison and M. Mojahedi, "Compact and broadband polarization beam splitter based on a silicon nitride augmented low-index guiding structure," Opt. Lett., vol. 41, no. 1, pp. 163-166, 2016.

[10] Z. Lian, P. Horak, X. Feng, L. Xiao, K. Frampton, N. White, J. A. Tucknott, H. Rutt, D. N. Payne, W. Stewart, and W. H. Loh, "Nanomechanical optical fiber," Opt. Express, vol. 20, no. 28, pp. 29386-29394, 2012.

[11] D. C. Zografopoulos, A. K. Pitilakis, and E. E. Kriezis, "Dual-band electro-optic polarization switch based on dual-core liquid-crystal photonic crystal fibers," App. Optics, vol. 52, no. 26, pp. 6439-6444, 2013.

[12] R. A. Hussein, M. F. O. Hameed, and S. S. Obayya, "Ultra-compact polarization splitter based on silica liquid crystal photonic crystal fiber coupler," ACES Journal, vol. 30, no. 6, pp. 599-607, 2015.

[13] J. Koo, J. Park, Y.-W. Song, S. Lee, K. Lee, J. H. Lee, "Fiber optic polarization beam splitter using a reduced graphene oxide-based interlayer," Opt. Mater., vol. 46, pp. 324-328, 2015.

[14] A. K. Pitilakis, D. C. Zografopoulos, and E. E. Kriezis, "In-line polarization controller based on liquid-crystal photonic crystal fibers," $J$. Lightwave Technol., vol. 29, no. 17, pp. 2560-2569, 2011.

[15] A. Lorenz, R. Schuhmann, and H.-S. Kitzerow, "Switchable waveguiding in two liquid-crystal-filled photonic crystal fibers," Appl. Opt., vol. 49, no. 20, pp. 3846-3853, 2010.

[16] A. Lorenz and H.-S. Kitzerow, "Efficient electro-optic switching in a photonic liquid crystal fiber", Appl. Phys. Lett., vol. 98, p. 241106, 2011.

[17] S. Ertman, A. H. Rodríguez, M. M. Tefelska, M. S. Chychłowski, D. Pysz, R. Buczyński, E. Nowinowski-Kruszelnicki, R. Dąbrowski, and T.R. Woliński, "Index guiding photonic liquid crystal fibers for practical applications", J. Lightwave Technol., vol. 30, no, 8, pp.1208-1214, 2012

[18] J. Sun and C. C. Chan, "Hybrid guiding in liquid-crystal photonic crystal fibers," J. Opt. Soc. Am. B, vol. 24, no. 10, pp. 2640-2646, 2007.

[19] D. C. Zografopoulos and E. E. Kriezis, "Tunable polarization properties of hybrid-guiding liquid-crystal photonic crystal fibers," J. Lightwave Technol., vol. 27, no, 6, pp.773-779, 2009.

[20] Z. Lian, M. Segura, N. Podoliak, X. Feng, N. White and P. Horak, "Nanomechanical optical fiber with embedded electrodes actuated by joule heating," Materials, vol. 7, pp. 5591-5602, 2014.

[21] N. Podoliak, Z. Lian, W. H. Loh, and P. Horak, "Design of dual-core optical fibers with NEMS functionality," Opt. Express, vol. 22, no. 1, pp. 1065-1076, 2014.

[22] S. Ertman, T. R. Wolinski, D. Pysz, R. Buczynski, E. NowinowskiKruszelnicki, and R. Dabrowski, "Low-loss propagation and continuously tunable birefringence in high-index photonic crystal fibers filled with nematic liquid crystals," Opt. Express, vol. 17, pp. 1929819310, 2009
[23] N. Podoliak, Z. Lian, M. Segura, W.H. Loh, P. Horak, "Electrostatic actuation of nanomechanical optical fibers with integrated electrodes," SPIE Proc., vol. 9128, p. 912802, 2014.

[24] O. Buchnev, N. Podoliak, M. Kaczmarek, N. I. Zheludev, and V. A. Fedotov, "Electrically controlled nanostructured metasurface loaded with liquid crystal: toward multifunctional photonic switch," Adv. Optical Mater., vol. 3, pp. 674-679, 2015.

[25] P. G. de Gennes and J. Prost, The Physics of Liquid Crystals, 2nd ed., Clarendon, Oxford,1993.

Nina Podoliak received the M.Sc. degree in theoretical physics from Kiev National University, Kyiv, Ukraine, in 2008, and the Ph.D. degree in physics from the University of Southampton, Southampton, U.K., in 2012. She has been a Post-Doctoral Research Fellow at the University of Southampton since 2012. Her current research interests include liquid crystal devices and applications, design and optimization of photonic systems, and modeling of light propagation in optical fibers and waveguides.

Peter Horak received the M.Sc. and Ph.D. degrees in theoretical physics from the University of Innsbruck, Innsbruck, Austria. Since 2001, he has been with the Optoelectronics Research Centre, University of Southampton, Southampton, U.K. $\mathrm{He}$ is interested in the theoretical and numerical investigation of photonic devices, including linear, nonlinear, and quantum optical effects. 